\title{
Assessment of cardiac function and subclinical atherosclerosis in children with systemic lupus erythematosus
}

Reham Wagdy ${ }^{1}$, Mohammed El Shafie², Mohie El-Kersh ${ }^{1}$

\author{
${ }^{1}$ Department of Paediatrics, Faculty of Medicine, Alexandria University, Alexandria, \\ Egypt \\ ${ }^{2}$ Department of Radiology, Faculty of Medicine, Alexandria University, Alexandria, \\ Egypt
}

Submitted: 10 November 2017

Accepted: 1 February 2018

Arch Med Sci

DOI: https://doi.org/10.5114/aoms/84970

Copyright @ 2021 Termedia \& Banach

\section{Abstract}

Introduction: Juvenile onset systemic lupus erythematosuS (JSLE) is an autoimmune disease associated with high cardiovascular morbidity. The purpose of the study was to assess cardiac function and subclinical atherosclerosis among children with SLE and to establish risk factors.

Material and methods: Data on thirty children with SLE followed up at Alexandria Main Children Hospital were collected and compared to thirty matched controls. The collected data were about demographics, systemic examination and laboratory findings. However, all participants were subjected to echocardiography, pulsed wave tissue Doppler (PWTD) and sonographic assessment of intima media thickness of the abdominal aorta (aIMT) and carotid arteries (cIMT).

Results: The patient group with mean age of $11.3 \pm 2$ years and mean duration of disease $3.2 \pm 2.3$ years included $63.3 \%(n=19)$ hypertensive cases with no heart failure. High cholesterolvalues were reported in $30 \%$. The main findings of the imaging studies of patients showed significantly low ejection fraction (EF) $(57.9 \pm 5.8, p<0.001)$ and low mitral annular velocities at septal and lateral walls at peak systole $(\mathrm{Sm})$ and peak early diastolic filling $(\mathrm{Em})(p<0.001)$. Also there were significantly high alMT $(1.1 \pm 0.3)$ and cIMT $(0.5 \pm 0.7)$ for patients. The duration of the disease and aIMT were significant risk factors that negatively affect Sm and Em of lateral wall $(p \leq 0.05)$.

Conclusions: In JSLE, impaired LV function and subclinical atherosclerosis were prevalent specially among children with longer disease duration which highlight the need for periodic checkup by ultrasonography and PWTD.

Key words: juvenile onset systemic lupus erythematosus, pulsed wave tissue doppler, intima media thickness, subclinical atherosclerosis.

\section{Introduction}

Juvenile onset systemic lupus erythematosus (JSLE) is a life-long autoimmune disease that can involve any organ system with a wide range of disease manifestations with significant morbidity and mortality.

The 10-year survival rates are now $>90 \%$, which is comparable with that of adults, because of major advances achieved in the field of pediatric rheumatology [1]. Those children are now surviving into adulthood and have to face many challenges imposed by their chronic illness. Car-
Corresponding author:

Reham Wagdy PhD

Department

of Paediatrics

Faculty of Medic

Alexandria University

Alexandria, Egypt

E-mail:dr_reham_wagdy@

yahoo.com 
diovascular morbidity and mortality are becoming major health concerns for those cases [2]. Cardiovascular involvement has a wide spectrum of presentation ranging from asymptomatic to severe overt conditions. In addition to pericardial, myocardial, valvular and rhythm disturbances a high incidence of coronary artery diseases (CAD) has become a recognized cause of mortality [2, 3]. Chronic inflammation and exposure to corticosteroids create perfect risk factors for endothelial injury and hence premature atherosclerosis [4]. Clinical cardiovascular involvement has been reported in many studies, but evidence of subclinical involvement is limited to a few of them [5].

\section{Material and methods}

The minimum sample size was calculated based on the smallest effect size between cases and controls regarding systolic and diastolic functions of the left ventricle reported by a certain study in 2003 [6]. It was equal to 50 divided into two equal groups. In order to increase the power of the study, the study was conducted on 60 children (30 with SLE and 30 controls).

This study has the case control design. It was performed between March 2012 and August 2012. The study included thirty children below the age of 16 years diagnosed with SLE and who were inactive at the time of the study (with normal inflammatory markers); the cases were followed up for more than 6 months at a pediatric nephrology clinic in line with the policy of the Main Children Hospital of Alexandria University. All active cases were excluded. The patient group at the time of diagnosis fulfilled the revised criteria of the American College of Rheumatology for the diagnosis of SLE; the criteria included all the old criteria such as photosensitivity, oral ulcers, and lupus nephritis while the updated changes in the immunologic disorder category consisted of removal of the LE cell item and addition of the anti-phospholipid antibody (aPL), anticardiolipin antibody ( $\mathrm{aCL}$ ), and lupus anticoagulant (LAC) items to positive anti-nuclear antibody and anti-double strand [7]. Another thirty children matched in age and sex were selected randomly after obtaining consent of caregivers. Those were children who presented to hospitals with acute minor illness (e.g. pneumonia, diarrhea) excluding patients with chronic medical diseases. Written informed consent was collected from patients' caregivers, and the local Ethics Committee approved the study protocol. Data of studied groups about medical history, physical examination, laboratory findings and electrocardiography were retrieved from hospital records while echocardiography and vascular sonography were performed for all.

\section{Echocardiography}

Two-dimensional echocardiography, M-mode, and Doppler flow modalities were applied to assess left ventricular function through measuring ejection fraction (EF), fractional shortening (FS) peak early diastolic filling velocity $(E)$, peak late diastolic filling velocity $(A)$, and $E / A$ ratio. The normal values for $E$ and $A$ velocities were $0.73 \pm 0.09$ and 0.38 $\pm 0.08 \mathrm{~m} / \mathrm{s}$ respectively [8]. Pulsed wave tissue Doppler (PWTD) recorded contraction and relaxation velocities of mitral annulus. The highest amplitude signal was evaluated in the apical four-chamber view; a 5-mm tissue Doppler sample volume was placed on the septal and lateral mitral annulus. The positive (Sm) wave represented ventricular systole while negative $(\mathrm{Em})$ and negative (Am) waves represented early and late diastole filling. Echocardiography was performed by a single person using a $5 \mathrm{MHz}$ transducer of Madison 990 echocardiography.

\section{Vascular ultrasonography}

Intimal medial wall thickness (IMT) measurements were obtained for the study and control groups using a Toshiba Nemio Ultrasonic scanner with a $7.5 \mathrm{MHz}$ transducer. Both carotid arteries were scanned $10 \mathrm{~mm}$ from the bifurcation of the common carotids. Intimal medial wall thickness of both right and left carotid arteries was measured three times each then the mean value was calculated to represent cIMT of the patient.

Meanwhile, the abdominal aorta was assessed at anterior and posterior walls and was examined distally until the aortic bifurcation $(8 \mathrm{~mm}$ thickness was the normal cut-off value for the IMT for the abdominal aorta) [9].

\section{Statistical analysis}

Data were fed to the computer and analyzed using IBM SPSS software package version 20.0. Comparisons between groups for categorical variables were assessed using the chi-square test (Fisher or Monte Carlo). The Mann-Whitney test was used to compare two groups for abnormally distributed quantitative variables. Student's $t$-test was used for normally distributed quantitative variables, to compare between two studied groups, Pearson's coefficient to correlate between two normally distributed quantitative variables, and Spearman's coefficient to correlate between two distributed quantitative abnormally variables. Significance of the obtained results was judged at the $5 \%$ level.

\section{Results}

Patients' age at the time of the study ranged from 6 to 15 years (mean: $11.3 \pm 2.6$ years) with a female to male ratio of $8 / 2$. The duration of 
disease ranged from 6 months to 9 years (mean: $3.2 \pm 2.3$ year) as shown in Table I. No significant difference for body mass index (BMI) was found between the participants. About $63.3 \%(n=19)$ of the SLE patients were hypertensive, eight had lower limb edema (26.6\%), four had sinus tachycardia, and none suffered from overt heart failure. At time of the study laboratory data indicated anemia among $20 \%$ of cases $(n=6)$ with insignificant variations in hemoglobin levels between studied groups. Proteinuria was evidenced among 3 cases. The mean values of serum triglyceride were significantly higher among cases compared to controls, while mean total serum cholesterol (TSC) and serum creatinine were within normal limits for all participants. However, high triglycerides were recorded among $36.3 \%$ ( $n=11$ cases) and high TSC was reported among 30\% ( $n=9$ cases). Renal biopsy specimens were analyzed for 26 patients and showed a wide range of pathologic changes. According to the modified WHO classification of glomerular lesions [10]: class I; minimal mesangial lupus nephritis, class II: mesangial proliferative lupus nephritis, class III; focal lupus nephritis, class IV; diffuse lupus nephritis, class V; membranous lupus nephritis and class IV; advanced sclerosis lupus nephritis. Class IV was observed in eleven pa tients, class II in eight, class III in 4 and class $V$ in 3 cases. All SLE patients were on a prednisone dose ranging between 0.2 and 2 (mean 0.8 ) $\mathrm{mg} / \mathrm{kg} /$ day.

\section{Cardiac function}

Although the mean EF and FS values of the patient group were within normal limits (EF: 57.9 \pm 5.8 and FS: $33.1 \pm 3.5)$, they were significantly lower than in controls (EF: $67.8 \pm 4.7$ and FS: 37.8 $\pm 3.4, p<0.001$ ). EF values were abnormally low in $6(20 \%)$ patients. Left ventricular diastolic functions were also impaired with significantly decreased E wave $(80.5 \pm 5.1$ vs. $92.6 \pm 7.6, p<0.001)$ and decreased E/A ratio $(1.5 \pm 0.1$ vs. $1.6 \pm 0.2$, $p=0.003)$, while peak $A$ velocity increased significantly (58 \pm 5.9 vs. $54.7 \pm 4.5, p=0.017)$ as shown in Table II. Meanwhile, PWTD findings showed that the mitral annular velocities at septal and lateral walls (Sm sep, Sm lat) during systole were significantly lower for the patient group $(10.3 \pm 1.8$ and $8.3 \pm 1.1)$ compared to controls $(11.8 \pm 1.3$ and $8.7 \pm 0.8, p<0.001)$. Also, mitral annular velocities during diastole (Sm sep, Sm lat, E sept, Elat, Em/ Am sept and Em/Am lat) were significantly lower for patients compared to controls $(p<0.001)$, as summarized in Table II. However, the E/Em ratio was significantly higher among patients. Our study revealed that some PWTD parameters (Sm lat, Sm sep and Em lat) showed significant negative correlations with the duration of the disease $(r=0.565, p=0.001 ; r=-0.466, p=00.9)$ while there was no significant correlation with total serum cholesterol or age, as shown in Table III. The hypertensive subgroup had significantly higher values of Em/Am ratio of the septal wall when compared to controls.

\section{Vascular sonography}

The mean values of alMT $(1.1 \pm 0.3)$ were significantly higher in children with SLE in comparison to controls $(0.7 \pm 0.1, p<0.00)$. About $63.3 \%$

Table I. Comparison between the two studied groups according to demographic data and laboratory profile of the studied groups

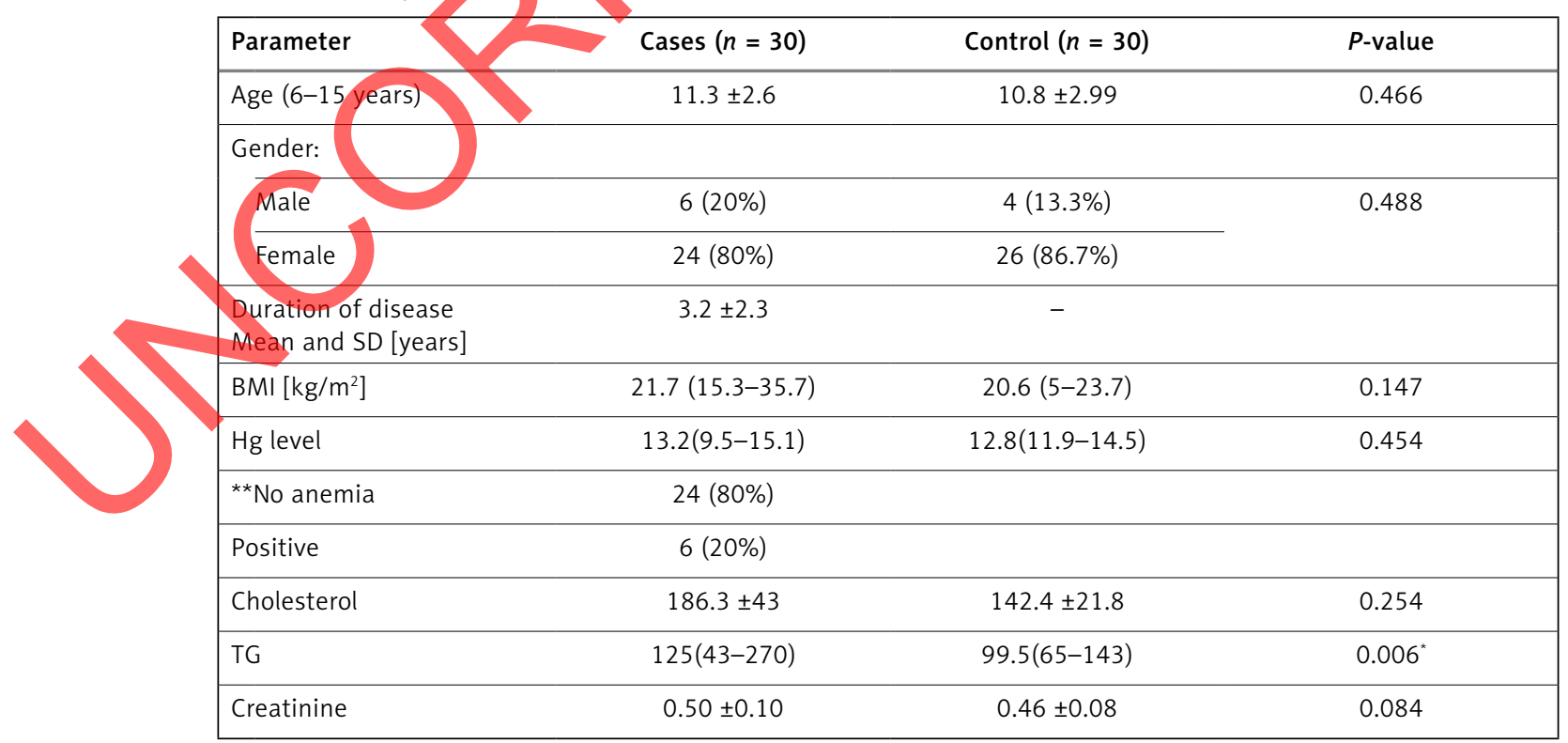

${ }^{*}$ Statistically significant at $p \leq 0.05 .{ }^{* *}$ Criteria of anemia according to age group; 5-12 years $\mathrm{Hg}<11.5 \mathrm{~g} / \mathrm{dl}, 12-14 \mathrm{years} \mathrm{Hg}<12 \mathrm{~g} / \mathrm{dl}$. Age $>15$-year girl $\mathrm{Hg}<12 \mathrm{~g} / \mathrm{dl}$, age $>15$-year boys $\mathrm{Hg}<13 \mathrm{~g} / \mathrm{dl}[$ [30]. 
Table II. Comparison between the two studied groups according to parameters of standard echocardiography, pulsed tissue Doppler and vascular sonography

\begin{tabular}{|c|c|c|c|}
\hline Parameter & Cases $(n=30)$ & Control $(n=30)$ & $P$-value \\
\hline \multicolumn{4}{|l|}{ Echocardiography } \\
\hline EF: & $57.9 \pm 5.8$ & $67.8 \pm 4.7$ & $<0.001^{*}$ \\
\hline$<55$ Abnormal & $6(20 \%)$ & $0(0 \%)$ & $0.024^{*}$ \\
\hline FS: & $33.1 \pm 3.5$ & $37.8 \pm 3.4$ & $<0.001^{*}$ \\
\hline$<29$ Abnormal & $4(13.3 \%)$ & $0(0 \%)$ & \\
\hline $\bar{E}$ & $80.5 \pm 5.1$ & $92.6 \pm 7.6$ & $<0.001^{*}$ \\
\hline$A$ & $58 \pm 5.9$ & $54.7 \pm 4.5$ & \\
\hline$E / A$ & $1.5 \pm 0.1$ & $1.6 \pm 0.2$ & .003 \\
\hline \multirow{2}{*}{\multicolumn{4}{|c|}{ PWTD (Mitral annulus): }} \\
\hline & & & \\
\hline Sm lat & $10.3 \pm 1.8$ & $11.8 \pm 1.3$ & $<0.001^{*}$ \\
\hline Em lat & $14.9 \pm 1.7$ & $18.2 \pm 18.2$ & $<0.001^{*}$ \\
\hline $\mathrm{Am}$ & $9.3 \pm 0.7$ & & 0.361 \\
\hline $\mathrm{Em} / \mathrm{Am}$ & $1.6 \pm 0.2$ & & $<0.001^{\star}$ \\
\hline $\mathrm{E} / \mathrm{Em}$ & $6.3 \pm 1.1$ & & $<0.001^{*}$ \\
\hline \multicolumn{4}{|l|}{ Septal wall MA: } \\
\hline Sm sep & $8.3 \pm 1.1$ & $8.7 \pm 0.8$ & 0.219 \\
\hline Em sep & $13.5 \pm 1.5$ & $15.2 \pm 0.6$ & $<0.001^{\star}$ \\
\hline Am sep & 9 & $8.2 \pm 0.5$ & $<0.001^{*}$ \\
\hline ES/AS & 1. & $1.9 \pm 0.1$ & $<0.001^{\star}$ \\
\hline cIMT: & & $(0.4-0.5)$ & $0.041^{*}$ \\
\hline Mean \pm SD & +07 & $0.5 \pm 0.1$ & \\
\hline Abnormal >0.55 & $(30 \%)$ & $0(0 \%)$ & \\
\hline alMT: & $5-1.7)$ & $(0.5-0.8)$ & $<0.001^{\star}$ \\
\hline Mean \pm SD & $1.1 \pm 0.3$ & $0.7 \pm 0.1$ & \\
\hline$>0.8$ Abnormal & 19 (63.3\%) & $0(0 \%)$ & \\
\hline
\end{tabular}

of cases $(n=19)$ had abnormal alMT $(>0.8 \mathrm{~mm})$. Table II. A significant positive correlation was observed between average of alMT and duration of the disease $(r=0.644, p<0.001)$ and hypertension ( $p=0.044)$ as summarized in Tables III and IV. Although the mean values of cIMT $(0.5 \pm 0.7)$ were significantly higher than in controls, high values were detected among 30\% of the patients. Lastly, there was significant negative correlation between the mitral annular velocities (Sm lat, Em) and alMT $(r=-0.657, p<0.001 ; r=-0.506, p<$ 0.004 respectively) which are displayed in Fig- ures $1 \mathrm{~A}$ and $\mathrm{B}$ quadrants. However, cIMT showed insignificant correlations with the same parameters, as shown in scatter plots in Figures $1 \mathrm{C}$ and D quadrants.

\section{Discussion}

Studying cardiovascular changes in children with SLE became challenging over the past decade. The current study focused on assessing the cardiac function of JSLE by different modalities of echocardiography and on detecting silent atherosclerotic changes by sonography. Also, the study assessed 
Table III. Correlation of risk factors versus tissue Doppler findings and abdominal aorta IMT in case group

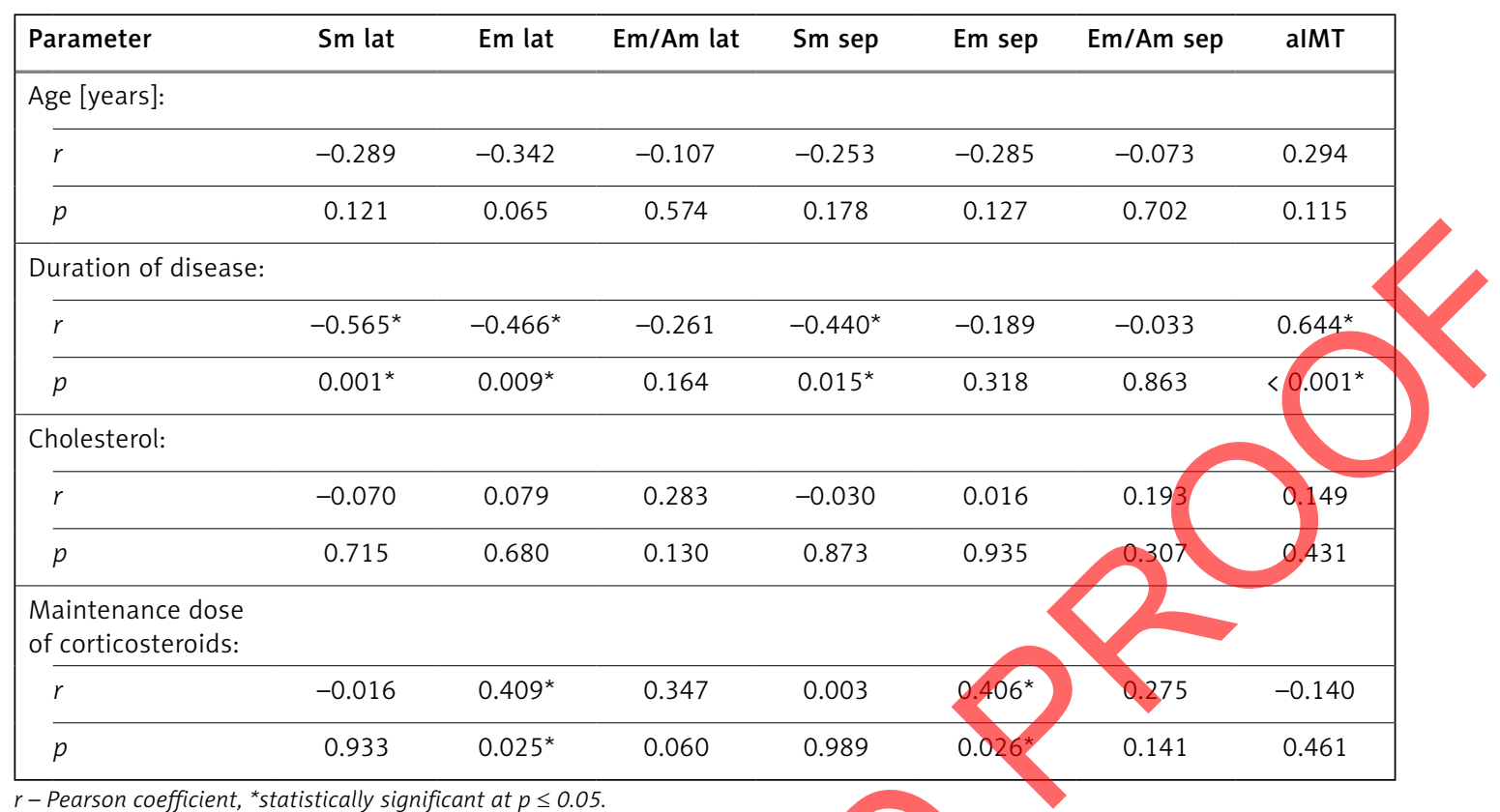

Table IV. Relation between hypertension with different echo and sonography parameters in case group

\begin{tabular}{|c|c|c|}
\hline Parameter & Hyperten & $P$-value \\
\hline & No $(n=11)$ & \\
\hline Sm lat & $10.5 \pm 1.4$ & 0.721 \\
\hline Em lat & $>15.1 \pm 1.7$ & 0.522 \\
\hline Em/Am lat & $1.7 \pm 0.2$ & 0.096 \\
\hline Sm sep & $8.4 \pm 1.2$ & 0.902 \\
\hline Em sep & $13.8 \pm 1.3$ & 0.164 \\
\hline Em/Am sep & $1.6 \pm 0.2$ & $0.010^{*}$ \\
\hline alMT & $1.1(0.7-1.7)$ & $0.044^{\star}$ \\
\hline
\end{tabular}

*Statistically significant at $p \leq 0.05$.

the risk factors of these cardiovascular changes as age of onset, duration of the disease, BMI, hypertension, dyslipidemia, renal pathology and dose of corticosteroid. Most of the enrolled patients (80\%) were female, similar to many reported studies [3, 5.6]. The age of onset of disease for most of our cases was above 5 years except two cases ( 3 and 4 years about $6.6 \%$ ), and this was found by another study [6] which reported that the occurrence of the disease below 5 years is rare. Body mass indices of patients were within the normal limits, similar to results of a Brazilian study [11] in 2014 where the median of patients was close to controls (21.74 vs. $21.43 \mathrm{~kg} / \mathrm{m}^{2}$ ). An Omani study that assessed BMI concluded that growth failure is an important determinant of disease damage, high steroid dose and longer disease duration [12].

Clinically, none of the cases were suffering from heart failure, and about two thirds developed hy- pertension. It is higher percent compared to that found by a previous study [13] which reported that about $25 \%$ of patients developed hypertension before the age of 18 years, but hypertension could be caused by renal disease and/or longterm high-dose corticosteroid. Captopril and atenolol were the used antihypertensive medications. In our study, renal involvement was $100 \%$ among patients who underwent renal biopsy $(n=26 / 30)$ with different classes of renal pathology and this may explain the high percentage of hypertensive patients. However, $57.8 \%$ of them were controlled. In concordance with some studies [6, 14] arrhythmia was positive among $10 \%$ of cases, which was documented by ECG to be sinus tachycardia.

Laboratory data at the time of the study revealed that about one third of patients had elevated levels of serum cholesterol and triglycerides; however, triglyceride levels were significantly 
A

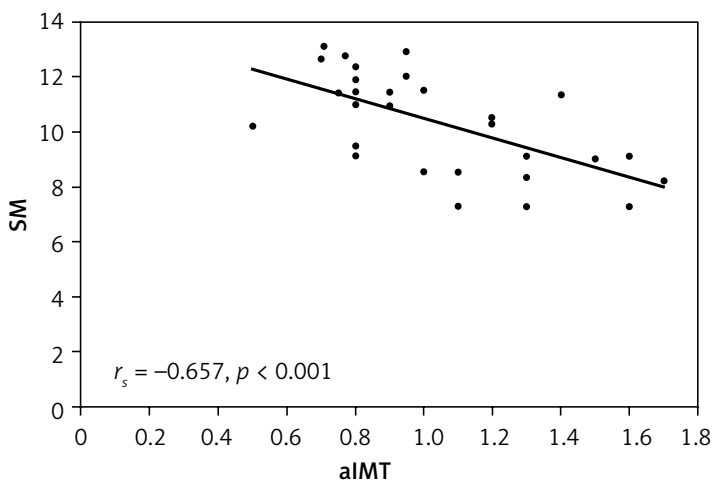

C

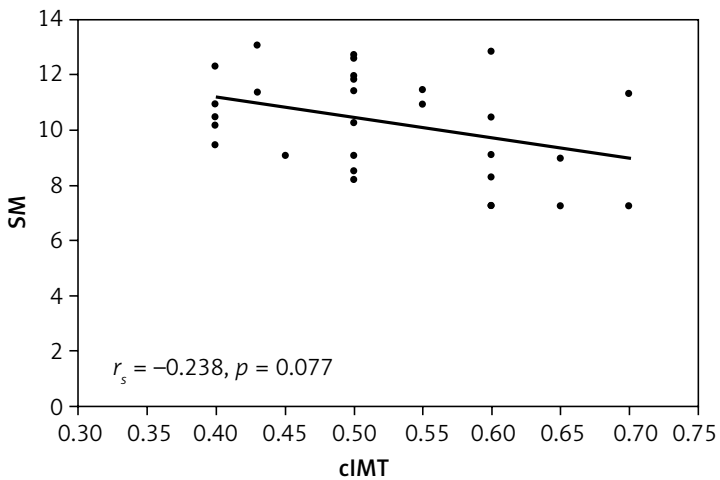

B

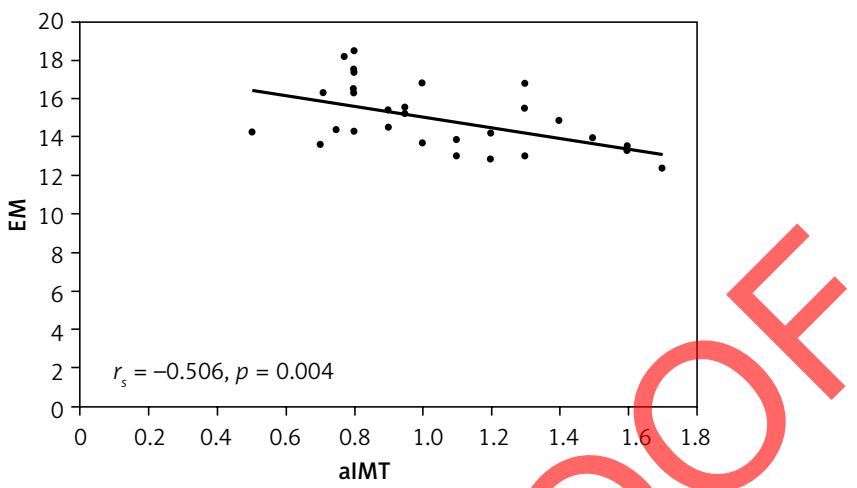

D

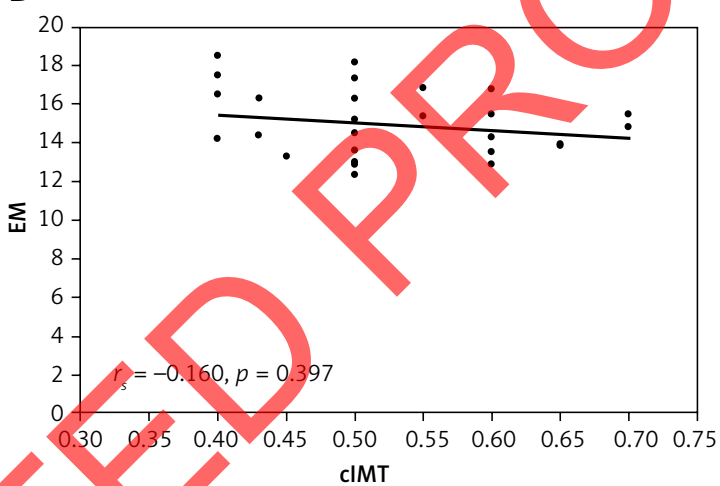

Figure 1. A - Correlation between aIMT and Sm lat in case group. B - Correlation between alMT and Em lat in case group. C - Correlation between cIMT with Sm lat in case group. D - Correlation between cIMT and Em lat in case group

higher in patients when compared to controls. This matched the pattern of dyslipidemia deation velocities of the myocardium through assessing mitral annular velocities. Therefore, PWTD scribed by other studies on adults with SLE [15] and children [16] which also reported high levels of LDL and low levels of HDL among the patients. That might be attributed to the used medications such as corticosteroids and atenolol (old $\beta$-blockers) which were reported by some studies to raise serum triglyceride [17]. In our study, renal function for all the patients was normal.

Echocardiographic evaluations of cardiac function were performed for the studied groups. Parameters of systolic function by standard modalities (EF and FS) were significantly lower in patients than controls. However, they were within normal and $20 \%$ of cases had abnormal EF. The diastolic function was impaired through significantly low $E$ wave in patients compared to controls while $A$ wave increased and E/A ratio decreased significantly but remained within the normal range. This was consistent with results obtained by many studies which explained the asymptomatic systolic and diastolic function impairment by lupus myocarditis (immunopathological changes of heart) $[6,15]$.

Recently, PWTD has been used as a sensitive method for evaluation of cardiac performance. It relies on determining the contraction and relaxcan evaluate global, regional and longitudinal myocardial contraction which may be involved subclinically. In contrast to EF, which evaluates the heart globally [18], the present study recorded regional defects in systolic and diastolic function of the lateral wall of the mitral annulus and impaired diastolic function of the septal wall. The average mitral annular velocity ( $\mathrm{Sm}$ lat) was significantly decreased in patients compared to controls while septal wall velocities (Sm sep) were insignificantly diminished. Moreover, diastolic indices (Em sep, Em late, Em/Am lat and E/Em lat) at septal and lateral walls were significantly lower in the patient group.

Due to the paucity of similar studies on children, we compared our results to similar studies performed on adults with SLE, which is in agreement with our results by finding significant impairment of regional wall function of the heart, whether systolic or diastolic indices [19]. Moreover, it matched the results of a Turkish study on children with familial Mediterranean fever (FMF) taking into consideration that both SLE and FMF are long lasting diseases based on immune dysregulation [20]. The asymmetry of the results for lateral wall compared to septal wall velocities may 
be explained by autoimmune inflammation in lupus carditis that was reported similarly in viral myocarditis studies to have predominance of lateral wall abnormalities (85\%) rather than septal wall (31\%) [21]. This could be attributed to the relation between inflammation and tissue perfusion in which the septal wall basically receives blood supply from right and left coronaries while the lateral wall is supplied only by the lateral circumflex [21]. In agreement with a recent study on lupus children, lateral wall regional changes were detected during active and inactive phases of the disease [22].

The current study correlated some risk factors with parameters PWTD. Duration of the disease was an important factor. The longer the duration of the disease, the greater was reduction in systolic and/or diastolic function of the heart, especially the lateral wall of the LV (Sm lat, Em lat, Sm sep). It was not only the longevity of the illness; our study referred also to age of onset of the disease, as $100 \%$ of patients who developed SLE below 5 years had abnormal diastolic function $(E / A<1$, $\mathrm{Em} / \mathrm{Am}<1$ ), although they had variable duration of illness $(5,6,7$ years).

On the other hand, maintenance dose of corticosteroid given during the inactive state was not correlated with regional cardiac wall velocities except early diastolic filling waves of lateral and septal walls were directly related. This may refer to the significant anti-inflammatory effect of steroids on improving diastolic wave velocities. Hypertension as a risk factor for cardiac changes was only correlated with Em/Am ratio of the septal wall; lower ratio than controls was a sign of impaired diastolic function. Small sample size might be the cause of absence of other correlations; the effect of hypertension deserves further investigations with larger samples of lupus hypertensive children. The current results on pediatric patients were consistent with another Egyptian study performed on adult SLE in 2013 [23].

Apart from cardiac involvement, acceleration of vascular changes is considered another serious complication for JSLE. The reason for premature atherosclerosis is unknown. The leading theory is that immune complex deposition over the vessels causes the initial intimal damage which triggers vascular change [24]. Lupus is now considered to be an independent risk factor for the development of atherosclerosis which enhances the development of coronary artery disease during adulthood.

The current study revealed that aIMT and CIMT were significantly higher among patients compared to controls. This matched some studies using IMT as a surrogate non-invasive early sonographic marker for atherosclerosis $[25,26]$.

Silent atherosclerotic changes were detected among two thirds of the patients by abnormal
alMT in comparison to one third of cases with abnormal cIMT. That was consistent with previous studies $[9,27]$ which concluded that the abdominal aorta is an earlier site of vascular changes in children. Our results revealed that hypertension was a potential risk factor for higher values of alMT matched with others [28]. A potent significant negative correlation was found between in aIMT and between Sm lat, Em lat obtained by tissue Doppler. The higher the values of alMT (atherosclerosis) the lower the cardiac indices (impaired function). This may be the first study to publish this correlation among pediatric patients. This was consistent with a Brazilian study in 2010 performed on adult females with subclinical atherosclerosis and they found that carotid IMT had negative correlations with tissue Doppler indices of diastolic function [29]. This refers to the effect of atherosclerosis on impairing cardiac function as it may enhance arterial stiffness of major arteries, such as the carotid artery and aorta, increasing afterload to the left ventricle, with a negative impact on diastolic function. This highlights the importance of screening and controlling subclinical vascular changes for better long-term cardiac performance [30].

In conclusion, juvenile onset SLE children are at high risk for silent impairment of cardiac function and for premature atherosclerosis. Regular follow-up of global cardiac function and regional myocardial performance are highly recommended not only by echocardiography but also with the tissue Doppler modality. Periodic check-up of IMT of the abdominal aorta is a good marker for detecting subclinical atherosclerosis, especially for children with long duration for the disease, hypertension, high maintained dose of corticosteroid and/or abnormal echocardiographic parameters of cardiac function.

\section{Acknowledgments}

We would like to thank the pediatric nephrology clinic team and staff.

\section{Conflict of interest}

The authors declare no conflict of interest.

\section{References}

1. Doria A, laccarino L, Ghirardello A, Zampieri S, Arienti S, Sarzi-Puttini P. Long-term prognosis and causes of death in systemic lupus erythematosus. Am J Med 2006; 119: 700-6

2. Barsalou J, Bradley TJ, Silverman ED. Cardiovascular risk in pediatric-onset rheumatological diseases. Arthritis Res Ther 2013; 15: 212

3. Mavrogeni S, Servos G, Smerla R, Markousis-Mavrogenis G, Grigoriadou G, Kolovou G. Cardiovascular involvement in pediatric systemic autoimmune diseases: the 
emerging role of noninvasive cardiovascular imaging Inflamm Allergy Drug Targets 2015; 13: 371-1.

4. Stojan G, Petri M Atherosclerosis in systemic lupus erythematosus. J Cardiovasc Pharmacol 2013; 62: 255-62.

5. Chung HT, Huang YL, Yeh KW, Huang JL. Subclinical deterioration of left ventricular function in patients with juvenile-onset systemic lupus erythematosus. Lupus 2015; 24: 263-72.

6. Günal N, Kara N, Akkök N, Çakar N, Kahramanyol O Akalýn N. Cardiac abnormalities in children with systemic lupus erythematosus. Turk J Pediatr 2014; 45: 301-5.

7. Hochberg MC. Updating the American College of Rheumatology revised criteria for the classification of systemic lupus erythematosus. Arthritis Rheum 2003; 40: 17-25.

8. Kimball TR, Meyer RA. Echocardiography. In: Moss and Adams' Heart Disease in Infants, Children and Adolescents. 6th ed. Allen HD (ed). Lippincott Williams and Wilkins, Philadelphia 2001; 252-62.

9. Davis PH, Dawson JD, Blecha B, Mastbergen RK, Sonka M. Measurement of aortic intimal-medial thickness in ad olescents and young adults. Ultrasound Med Biol 2010; 36: 560-5.

10. Weening JJ, D'Agati VD, Schwartz MM, et al. The classification of glomerulonephritis in systemic lupus erythematosus revisited. J Am Soc Nephrol 2004; 2: 241-50.

11. Sinicato NA, Postal M, Peres FA, et al. Obesity and cytokines in childhood-onset systemic lupus erythematosus. J Immunol Res 2014; 2014: 162047.

12. Abdalla E, Jeyaseelan L, Ullah I, Abdwani R. Growth pattern in children with systemic lupus erythematosus. Oman Med I 2017; 32: 284-90.

13. Ostrove BE, Eichenfild AH, Goldsmith DP, Kaplan B, Atheya B. Hypertension in children with systemic lupus erythematosus. Semin Arthritis Rheum 1989; 19: 90-8.

14. Ahmed A, El Shama M. Asymptomatic cardiac involvement in children with systemic lupus erythematosus J Med Sci 2006; 6: 944-9.

15. Yuan J, Li LI, Wang Z, Song W, Zhang Z. Dyslipidemía in patients with systemic lupus erythematosus: association with disease activity and B-type natriuretic peptide levels. Biomed Rep 2016; 4: 68-72.

16. Ortiz TT, Terreri MT, Caetano M, et al. Dyslipidemia in pediatric systemic lupus erythematosus: the relationship with disease activity and plasma homocysteine and cysteine concentrations. Ann Nutr Metab 2013; 63: 77-82.

17. Nandeesha H, Pavithran P, Madanmohan T. Effect of antihypertensive therapy on serum lipids in newly diagnosed essential hypertensive men. Angiology 2009. 60:217-20.

18. Kadappu KK, Thomas L. Tissue Doppler imaging in echocardiography: value and limitations. Heart Lung Circulation 2015; 24: 224-33.

19. Elnady BM, Abdelghafar AS, Khalik ES, et al. The implication of tissue Doppler echocardiography and cardiopulmonary exercise in early detection of cardiac dysfunction in systemic lupus erythematosus patients. Eur J Rheumatol 2016; 3: 109-17.

20. Ceylan Ö, Özgür S, Örün UA, Doğan V, Yılmaz O, Keskin M. Assessment of left ventricular functions with tissue Doppler, strain, and strain rate echocardiography in patients with familial Mediterranean fever. Anatol J Cardiol 2015; 15: 663-8.

21. Li YD, Hsiao FT, Lai CP, Chen CW. Acute viral myocarditis mimicking ST elevation myocardial infarction: manifestation on cardiac magnetic resonance. Case report. Acta Cardiol Sin 2010; 26: 44.
22. Khositseth A, Prangwatanagul W, Tangnararatchakit K, Vilaiyuk S, Su-Angka N. Myocardial performance index in active and inactive paediatric systemic lupus erythematosus. Clin Exp Rheumatol 2017; 35: 344-50.

23. Allama N, Darweech HE, Hamadanallha N, Ashour $Z$ Evaluation of left ventricular myocardial function in Egyptian patients with systemic lupus erythematosus: tissue Doppler study and its relation to disease activity the Egyptian. Rheumatologist 2013; 35: 217-23.

24. Zeller CB, Appenzeller S. Cardiovascular disease in systemic lupus erythematosus. The role of traditional and lupus related risk factors. Curr Cardiol Rev 2008; 4: 116-22.

25. Quinlan C, Kari J, Pilkington C, Deanfield J, Shroff R, Marks ST The vascular phenotype of children with systemic lupus erythematosus. Pediatr Nephrol 2015; 30 1307-16.

26. Sozeri B, Deveci M, Dincel N, Mir S. The early cardiovascular changes in pediatric patients with systemic lupus erythematosus. Pediatr Nephrol 2013; 28: 471-6.

27. Järvisalo MJ, Jartti L, Salonen KN, trjala K, Rönnemaa T, Hartiala JJ. Increased aortic intima-media thickness a marker of preclinical atherosclerosis in high-risk children. Circulation 2001; 29: 43-7.

28. Boros CA, Bradley TJ, Cheung MM, et al. Early determinants of atherosclerosis in pediatric systemic lupus erythematosus. Clin Exp Rheumatol 2011; 29: 575-81.

29. Garcia MMO, Rodrigues MG, Neto JAD, Correia LC. Influence of subclinical atherosclerosis on diastolic function in individuals free of cardiovascular disease. Arq Bras Cardiol 2011; 95: 473-8.

O. WHO, UNICEF, UNU. Iron deficiency anemia: assessment, prevention and control, a guide for programme managers. Geneva, World Health Organization, 2001. 